\title{
TITLE:
}

\section{Myeloid Derived Suppressor Cells in Lung Adenocarcinoma}

\section{AUTHORS:}

Laura G. Rico ${ }^{1}$, Andrés Aguilar Hernández ${ }^{2}$, Michael D. Ward ${ }^{3}$, Jolene A. Bradford ${ }^{3}$, Jordi Juncà $^{4}$, Rafael Rosell ${ }^{2,4^{*}}$, and Jordi Petriz ${ }^{1 *}$

${ }^{1}$ Functional Cytomics Group, Institut de Recerca contra la Leucèmia Josep Carreras, IJC Campus ICO-Germans Trias i Pujol, Institut Germans Trias i Pujol (IGTP), Universitat Autònoma de Barcelona, UAB, 08916 Badalona, Barcelona, Catalonia, Spain

${ }^{2}$ Quirón Dexeus University Hospital, Dr Rosell Oncology Institute, Barcelona, Spain

${ }^{3}$ Thermo Fisher Scientific, Eugene, Oregon, USA

${ }^{4}$ Institut Català d'Oncologia, Hospital Germans Trias i Pujol (HGTiP), Badalona, Spain

\section{KEYWORDS:}

Flow cytometry, PD-L1, immunotherapy, MDSCs

WORD COUNT: 503

\section{CORRESPONDING AUTHOR:}

Jordi Petriz, PhD

Josep Carreras Leukaemia Research Institute

Crta. de Can Ruti, Camí de les Escoles s/n. Edifici IJC

08916 Badalona (Barcelona), Spain

Email: jpetriz@carrerasresearch.org

Rafael Rosell, MD

Molecular and Cellular Oncology Laboratory

Germans Trias i Pujol Research Institute and Hospital (IGTP)

Crta. de Can Ruti, Camí de les Escoles s/n.

08916 Badalona (Barcelona), Spain

Email: rrosell@iconcologia.net

\section{CONFLICT OF INTEREST:}

M.D.W. and J.A.B. work for Thermo Fisher Scientific, which is in the business of selling flow cytometers and flow cytometry reagents. 


\section{AUTHOR CONTRIBUTION STATEMENT:}

L.G.R., and J.P. performed the experiments. L.G.R., A.A.H, M.D.W., J.A.B., J.J., R.R. and J.P performed data interpretation, and drafted the manuscript. R.R. and J.P conceived the study, participated in its design, conducted most experiments, and contributed the manuscript equally $\left(^{*}\right)$. 


\begin{abstract}
Immunohistochemicaland flow cytometric assessment of PD-L1 expression on tumor cells and immunecells might suppose a great benefit to identify patients undergoing immunotherapies. However, the use of different techniques and antibodies cancomplicate valid comparisons between results obtained in different trials, and even more because of small biopsy specimens can result infalse-negative PD-L1 evaluation. Herein we describe the accurate identification of PD-L1 on peripheralblood myeloid derived suppressor cells (MDSCs) in such a case.
\end{abstract}


Dear Editor:

PD-L1 expression on tumor cells and immune cells could predict response to immunotherapy ${ }^{1}$. However, tiny biopsy samples can result in false-negative PD-L1 evaluation ${ }^{2}$. Herein, we describe the accurate identification of PD-L1 on peripheral blood myeloid derived suppressor cells (MDSCs).

A 72-year-old male, never-smoker, was diagnosed with recurrent lung adenocarcinoma, PD-L1 negative (22C3), without actionable mutations. The patient was treated with nivolumab, and paclitaxel. After progression, he was re-treated with nivolumab plus glutaminase inhibitor until persistence of adrenal and distant lymph node metastasis was observed. Atezolizumab plus T cell immunoglobulin mucin-domain containing-3 (TIM3) inhibitor was administered as third line therapy. The patient completed 11 cycles. Afterwards, the left adrenal gland was irradiated and a muscle metastasis in the left gluteus was resected. Targeted Next Generation Sequencing (NGS) in metastatic tissue and blood were negative for actionable alterations. The patient received further treatment with a novel anti-PD-L1 (PDR001) combination. However, further progression was identified with widespread bone and lymph node metastases (More details in Supplementary Figure 1). Peripheral blood samples were serially collected before and after immunotherapy for the assessment of MDSCs and PD-L1 expression by multi-color flow cytometry.

Peripheral blood smear analysis showed mostly neutrophils and monocytes, and lymphocytes were clearly reduced (Supplementary Figure 2). Hematology analysis showed a decreased number of lymphocytes (5.5\%), and a marked increase in monocytes $(13 \%)$ and neutrophils (80.2\%). At initial assessment, red-cell count, hemoglobin and hematocrit showed decreased concentrations. White-cell analysis by multi-color flow 
cytometry showed that PD-L1 was dramatically increased after Phorbol 12-myristate 13acetate (PMA) stimulation. Peripheral blood cells were mostly PD-L1+ MDSCs $(81.50 \%$ polymorphonuclear PMN-MDSCs and 7.74\% monocytic M-MDSCs) (Figure 1).

Surprisingly, non-stimulated cells expressed undetectable levels of PD-L1, as shown by the flow cytometry method when DMSO was used. This suggests that PD-L1 displays some variation of spatial conformation ${ }^{3}$ in response to PMA stimulation, which may confer a critical enhancement in binding affinity.

Eliminating MDSCs by promoting PD-L1 stabilized unfolded states on both PMN- and MMDSCs could improve immunotherapy efficacy. High frequency of PMN-MDSCs ${ }^{4}$ is an adverse prognostic factor in lung ${ }^{5}$ and head and neck cancer ${ }^{6,7}$. This conformational change may be associated with a PD-L1 immunoregulatory mechanism that affects therapies targeting the PD-1/PD-L1 checkpoint. Conformational changes, accumulation, expansion, and survival of MDSCs require research for PD-L1 unfolding. Monitoring peripheral blood MDSCs and identification of drugs with PMA-like effects is highly warranted. In view of these conformational properties, accumulation, expansion and survival of pathological immunosuppressive MDSCs could help to better understand and overcome the mechanisms of immunotherapy resistance, by developing new treatment strategies aimed at promoting PD-L1 stabilized unfolded states. Altogether, our biological findings can indicate the clinical relevance of peripheral blood MDSCs assessment and immunosuppressive pattern, as well as the need to find compounds that by themselves exhibit a PMA-like effect aimed to abrogate MDSCs. The feasibility of determining PD-L1 expression on MDSCs and its potential use as a biomarker to determine treatment response is promoting new ways to study cancer, particularly in the promising area of immunotherapy. 
Figure 1

Figure 1. Evaluation of PD-L1 expression and clustering analysis in terms of cell number and antigenic density comparisons across immunotherapy.

Evaluation of PD-L1 fold-increase of a 72-year-old male, a lifetime non-smoker, diagnosed with lung adenocarcinoma in 2014 . Peripheral blood was stimulated for 10 minutes at $37^{\circ} \mathrm{C}$ with PMA. Fold-change was calculated as the ratio between stimulated PD-L1+ MDSCs and non- stimulated PD-L1+ cells. The PD-L1+ population was calculated over the total number of acquired leukocytes. Changes in PD-L1 expression after Phorbol 12-Myristate 13-Acetate (PMA) treatment found negative to positive conversion, from 1 (Panel $A$ ) to 191-fold increase after stimulation (Panel B), suggesting that fold-change in PD-L1 appears to have an important regulatory factor that may relate to differences in underlying mechanisms within patients. This variability may help to explain the different response to targeted immunotherapy against PD-L1, also suggesting that phenotypic characterization is not sufficient to identify MDSCs and an additional proof of the immunosuppressive function is needed. PD-L1 appears to be rapidly translocated to the cell surface in response to PMA stimulation. Although the mechanisms by which PD-L1 translocates to the cell surface are still unclear, they may be related to mechanisms that support tumor progression and therapeutic response of cancer. Important research must be done to elucidate the mechanism by which PD-L1 is translocated and the clinical relevance of those patients with higher PD- L1 fold-change. Future experiments will attempt to further dissect to what degree stimulation increases PD-L1 expression on MDSCs and how this information can be translated into benefit for immunotherapy. The feasibility of determining PD-L1 expression on MDSCs and its potential use as a biomarker to determine treatment response is promoting new ways to study cancer, particularly in the promising area of immunotherapy.

Human peripheral blood specimens used in this study were collected with EDTA anticoagulant. For functional analysis, no-lyse no-wash (NLNW) procedures were applied. PE-PD-L1 staining was combined with APC-CD11b, PE-Cy7-CD33, and FITC-HLA-DR to detect MDSCs using NLNW methods. PD-L1 expression was studied in a total of $n=5$ samples, before and after immunotherapy (on day 0,1, 4, 6, and 8) with and without blood stimulation. For stimulation, peripheral blood cells were treated with PMA for 10 minutes in $37^{\circ} \mathrm{C}$ water bath. DyeCycle Violet (DCV) was used to discriminate nucleated cells from erythrocytes, platelets and debris. Violet side scatter (VSSC) was collected from the 405 $\mathrm{nm}$ laser with a $405 / 10$ bandpass filter. For VSSC, the accuracy of the height parameter is better preserved than for $488 \mathrm{~nm}$ blue SSC because the hemoglobin absorption of coincident erythrocytes reduces its highest signal relative to the leukocytes. Samples were acquired using the Attune ${ }^{\mathrm{TM}} \mathrm{NxT}$ Flow Cytometer (Thermo Fisher).

Merging all together into a single FCS file using a process called concatenation, peripheral blood samples obtained before and after immunotherapy treatment were effectively compared. Dimensionality reduction was performed on the concatenated data set. After PMA stimulation, PD-L1+ cells were displayed based on the expression of 6 markers using $\mathrm{t}-\mathrm{SNE}$ (t-distributed stochastic neighbor embedding) visualization. The axes (t-SNE1 and tSNE2) show dimensionless values that were assigned to individual cells by the t-SNE algorithm, which places PD-L1+ MDSCs that have similar expression profiles close to one another. In Panel C, the intensity of the coloring is relative to the cell density of PD-L1+ MDSCs. In Panel D, the intensity of the coloring is relative to the expression of PD-L1+ 
MDSCs. MDSCs from pre- and post-immunotherapy have similar cell density and expression patterns that identify them as well conserved entities across biologic therapy. 


\section{References}

1. Hirsch FR, McElhinny A, Stanforth D, Ranger-Moore J, Jansson M, Kulangara K, Richardson W, Towne P, Hanks D, Vennapusa B, Mistry A, Kalamegham R, et al. PD-L1 Immunohistochemistry Assays for Lung Cancer: Results from Phase 1 of the Blueprint PD-L1 IHC Assay Comparison Project. J Thorac Oncol 2017;12:208-22.

2. Kitazono S, Fujiwara Y, Tsuta K, Utsumi H, Kanda S, Horinouchi H, Nokihara H, Yamamoto N, Sasada S, Watanabe S, Asamura H, Tamura T, et al. Reliability of Small Biopsy Samples Compared With Resected Specimens for the Determination of Programmed Death-Ligand 1 Expression in Non--Small-Cell Lung Cancer. Clin Lung Cancer 2015;16:385-90.

3. Pascolutti R, Sun X, Kao J, Maute RL, Ring AM, Bowman GR, Kruse AC. Structure and Dynamics of PD-L1 and an Ultra-High-Affinity PD-1 Receptor Mutant. Structure 2016;24:1719-28.

4. Tcyganov E, Mastio J, Chen E, Gabrilovich DI. Plasticity of myeloid-derived suppressor cells in cancer. Curr Opin Immunol 2018;51:76-82.

5. Barrera L, Montes-Servin E, Hernandez-Martinez JM, Orozco-Morales M, Michel-Tello D, Morales-Flores RA, Flores-Estrada D, Arrieta O. Levels of peripheral blood polymorphonuclear myeloid-derived suppressor cells and selected cytokines are potentially prognostic of disease progression for patients with non-small cell lung cancer. Cancer Immunol Immunother 2018;67:1393-406.

6. Lang S, Bruderek K, Kaspar C, Hoing B, Kanaan O, Dominas N, Hussain T, Droege F, Eyth C, Hadaschik B, Brandau S. Clinical Relevance and Suppressive Capacity of Human Myeloid-Derived Suppressor Cell Subsets. Clin Cancer Res 2018;24:4834-44.

7. Lee HT, Lee SH, Heo YS. Molecular Interactions of Antibody Drugs Targeting PD-1, PD-L1, and CTLA-4 in Immuno-Oncology. Molecules 2019;24. 


\section{ACKNOWLEDGEMENTS}

We thank CERCA Programme/Generalitat de Catalunya and The Josep Carreras Foundation for institutional support. The authors are very grateful to Sara Vergara and Minerva Raya, for kindly providing samples used in this study. The authors are also very grateful for their advice and technical support to Sergio Ramón, Víctor Querol, Clara Streiff, Paola Paglia, and Lluís Sainz from Thermo Fisher for all his comments and discussions on earlier work in this research field. Jordi Petriz also acknowledges the financial support from The Obra Social La Caixa. 


\section{Supplementary Appendix}

This appendix has been provided by the authors to give readers additional information about their work. 


\section{Supplemental Appendix}

\section{Table of Contents}

List of Investigators

Supplemental Patient Information

Supplemental Methods

Fig.S1. PET-CT scan showing wide-spread metastasis involving bones, abdominal lymph nodes and mediastinal lymph nodes.

Fig.S2. Peripheral blood smear analysis showing mostly neutrophils and monocytes, and lymphocytes were clearly reduced. 


\section{List of Investigators}

Laura G. Rico, Andrés Aguilar Hernández, Michael D. Ward, Jolene A. Bradford, Jordi Juncà, Rafael Rosell, and Jordi Petriz 


\section{Supplemental Patient Information}

A 72-year-old male, a lifetime non-smoker, was diagnosed with lung adenocarcinoma in May 2014. An upper left lobectomy was performed in June 2014 revealing a mass of 1.5 $\mathrm{cm}$ with visceral pleural invasion. It was staged as IB (pT2N0M0). No adjuvant chemotherapy was prescribed. On July 2015 , he developed a sudden onset of hoarseness. Unilateral vocal cord paralysis was noted from laryngoscopy. PET CT scan revealed upper left pleural and mediastinal hypermetabolic area and a left adrenal metastasis. Molecular testing was negative for actionable mutations (EGFR, ALK, ROS1, RET fusions, MET exon 14 skipping mutations by RT-PCR), as well as for KRAS and BRAF mutations. PD-L1 by immunohistochemistry assay was negative PD-L1 expression was assessed during screening at two central laboratories with the PD-L1 IHC 22C3 pharmDx assay (Agilent Technologies, Carpinteria, CA, USA) and measured in formalinfixed tumour samples obtained by core-needle or excisional biopsy of a tumour lesion or from tissue resected at or after the time metastatic disease was diagnosed.

. Brain MRI was normal. He began chemotherapy with carboplatin and pemetrexed. Stereotactic radiotherapy for the pleural and adrenal lesions was recommended, but was not performed, since the patient obtained complete response with chemotherapy. After 6 cycles of chemotherapy, he continued with pemetrexed alone, as maintenance therapy. However, in July 2016, after 9 cycles of pemetrexed. Recurrence was observed in both the pleural mediastinal area, as well as in the left adrenal gland. He received $52 \mathrm{~Gy}$ in the mediastinal lung area and 42Gy in the left adrenal gland. In October 2018, a right femur metastasis appeared, which was treated with radiotherapy. In January 2017, further tumor progression was observed in the left adrenal gland and in distant lymph nodes. Nivolumab plus paclitaxel was started as a second line of systemic therapy. He continued the treatment until July 2017, when further tumor progression was seen. The patient started a clinical trial with a combination of nivolumab plus glutaminase inhibitor until January 2017, when persistence of the adrenal metastasis was observed, as well as, further tumor progression in lymph nodes. The patient started a third line of treatment with atezolizumab plus T cell immunoglobulin mucin-domain containing-3 (TIM3) inhibitor. The patient completed 11 cycles without side effects. In October 2018, he completed a second irradiation of the left adrenal gland. A muscle metastasis in the left gluteus was resected in September 2018. NGS-based tumor profiling was performed in the blood and tumor tissue without identifying actionable alterations (AKT1 ALK BRAF DDR2 EGFR ERBB2 ERBB3 ESR1 KIT KRAS MAP2K1 NRAS NTRK1 PDGFRA PIK3CA PTEN RAF1 ROS1). The patient was treated in a new clinical trial with an anti-PD-L1 (PDR001) plus canakinumab, however, after 5 cycles, the patient showed progression with a lymph node of the left groin infiltrating the skin. In July 2019 the patient was hospitalized. The brain MRI revealed a sole brain occipital metastasis. The Positron emission tomography-computed tomography (PET-CT) scan showed wide-spread metastasis involving bones, abdominal lymph nodes and mediastinal lymph nodes.

On examination, the patient appeared well. His temperature was $36.8^{\circ} \mathrm{C}$, his pulse was 80 beats/minute and blood pressure was $110 / 70 \mathrm{~mm} \mathrm{Hg}$. A right lateral cervical soft lymph node was palpable and the left inguinal lymph node was erythematous and involved the skin. The results of renal liver function tests were normal. Blood levels of electrolytes show 
hyponatremia. The patient started treatment with gemcitabine and pembrolizumab on July $5^{\text {th }}$. 


\section{Supplemental Methods}

Cell morphology. Blood smears were prepared, dried, fixed, and stained by May Grünwald-Giemsa stain. Photomicrographs were taken on a Olympus BX53 light microscope equipped with an Olympus DP21 digital camera. The cellSens software was used to capture and adjust the images accordingly.

Flow cytometry. Briefly, EDTA-anticoagulated blood samples were obtained the day before immunotherapy administration (PRE-OT) and 1 hour after immunotherapy (POSTOT). Samples were prepared and analyzed immediately after collection. The patient enrolled in this study provided an informed consent in accordance with the Declaration of Helsinki. All procedures were in accordance with the internal protocols of the laboratory, which were authorized by the HGTiP Ethical Committee. In order to minimize sample perturbation, samples were prepared using unlysed whole blood and no-lyse no-wash protocols. Two aliquots containing $100 \mu \mathrm{l}$ whole blood were incubated with $10 \mu \mathrm{l}$ FBS and $2 \mu$ Vybrant $^{\mathrm{TM}}$ DyeCycle $^{\mathrm{TM}}$ (DCV) Violet Stain (Thermo Fisher) $(50 \mu \mathrm{M})$ for 10 minutes at $37^{\circ} \mathrm{C}$ in a dedicated water bath. DCV was used to discriminate nucleated cells from erythrocytes and debris. For stimulation, one aliquot was treated with $1.63 \mu \mathrm{M}$ Phorbol $12-$ myristate 13-acetate (PMA, Sigma Aldrich) for 5 minutes at $37^{\circ} \mathrm{C}$ in a dedicated water bath. In parallel, the second aliquot was treated with DMSO, used as solvent for PMA (non-stimulated sample). In order to identify MDSCs, PE-conjugated PD-L1 monoclonal antibody (eBioscience ${ }^{\mathrm{TM}}$ ) staining was combined with APC-CD11b (Invitrogen ${ }^{\mathrm{TM}}$ ), PE-Cy7CD33 (eBioscience ${ }^{\mathrm{TM}}$ ), and FITC-HLA-DR (Invitrogen ${ }^{\mathrm{TM}}$ ). Antibodies were added at a concentration of $2,5 \mu \mathrm{g} / \mathrm{mL}$ and incubated for $20 \mathrm{~min}$ at room temperature. Prior to acquisition, stained cells were diluted in $1000 \mu \mathrm{L}$ HBSS. 7-AAD $(1.5 \mu \mathrm{M})$ was added to exclude necrotic cells. All cell measurements were done on the Attune ${ }^{\mathrm{TM}} \mathrm{NxT}$ Flow Cytometer (Thermo Fisher). Samples were acquired at $25 \mu \mathrm{L} / \mathrm{min}$ sample rate, and a minimum of 20,000 live cells per sample was collected and analyzed. Thresholding was empirically set to identify nucleated cells and to eliminate erythrocytes, platelets and debris using a V-SSC vs. DCV dual parameter plot. SSC was detected using the $405 \mathrm{~nm}$ violet laser with a 405/10nm BP filter. DCV was excited at 405nm and its emission was collected with a 440/50 BP filter. FITC was excited with 488nm blue laser and its emission was collected with 530/30 BP filter. PE, 7-AAD, and PE-Cy7 were excited with $561 \mathrm{~nm}$ yellow/green laser and its emission was collected with a 585/16 BP filter (PE), a 620/15 BP filter (7-AAD), and a 780/60 BP filter (PE-Cy7). APC was excited using the $637 \mathrm{~nm}$ red laser and its emission was collected with a 670/14 BP filter. FSC and SSC were displayed on a linear scale. DCV, FITC, PE, 7-AAD, PE-Cy7, and APC were displayed on a logarithmic scale. To visualize the PD-L1 data, which clustered into multiple clusters, tSNE was used. Data were analyzed using FlowJo version 10.5.3 (FlowJo LLC, Ashland, OR, USA). 


\section{Supplemental Figure 1}

\section{METASTATIC LESIONS SEEN ON PET-CT}

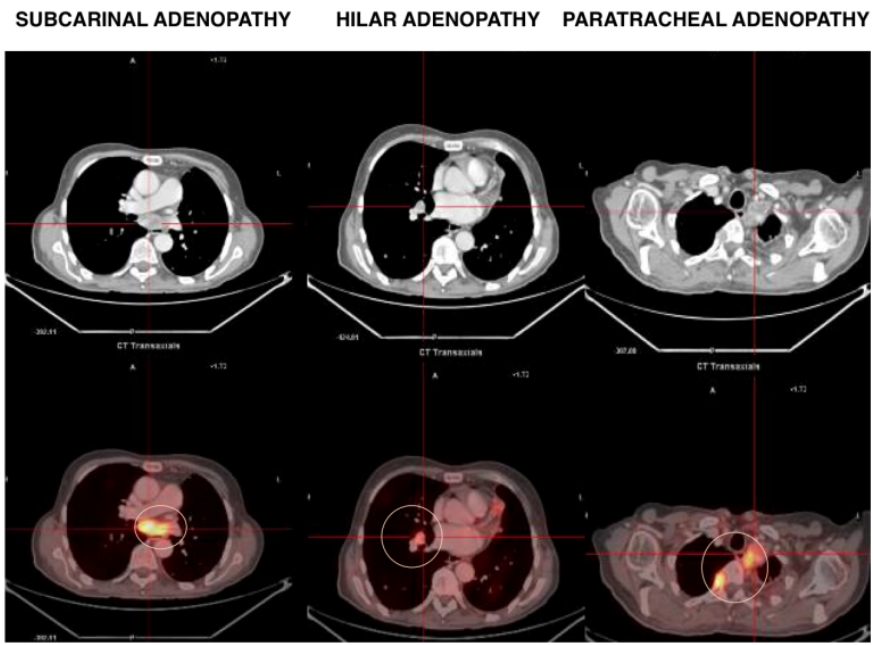

INGUINAL ADENOPATHY PARAVERTEBRAL NODE

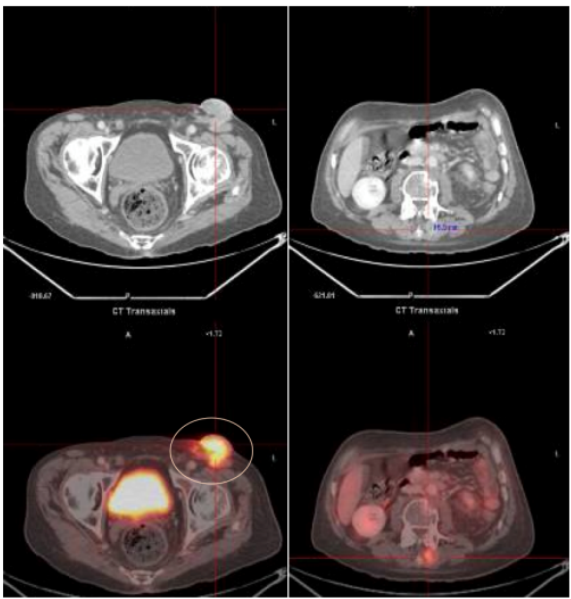

PET-CT scan showing wide-spread metastasis involving bones, abdominal lymph nodes and mediastinal lymph nodes.

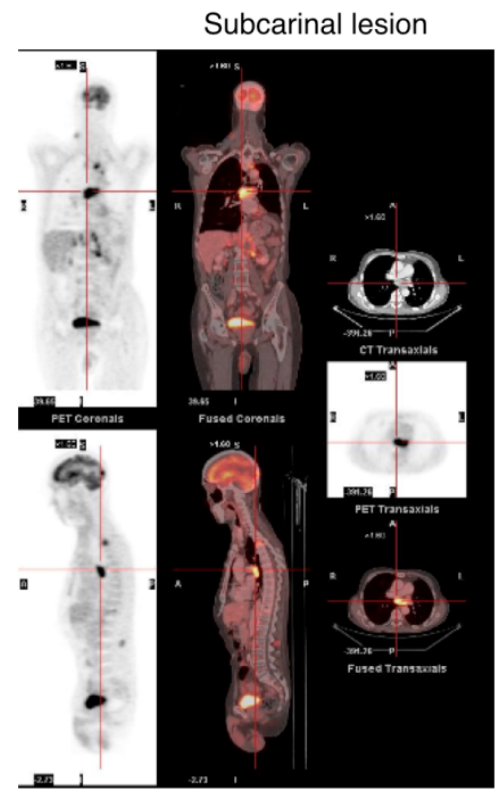

Laterocervical, paratracheal, hilar, subcarinal, paraaortic adenopathies

Peripheral blood smear analysis showing mostly neutrophils and monocytes, and lymphocytes were clearly reduced. 


\section{Supplemental Figure 2}
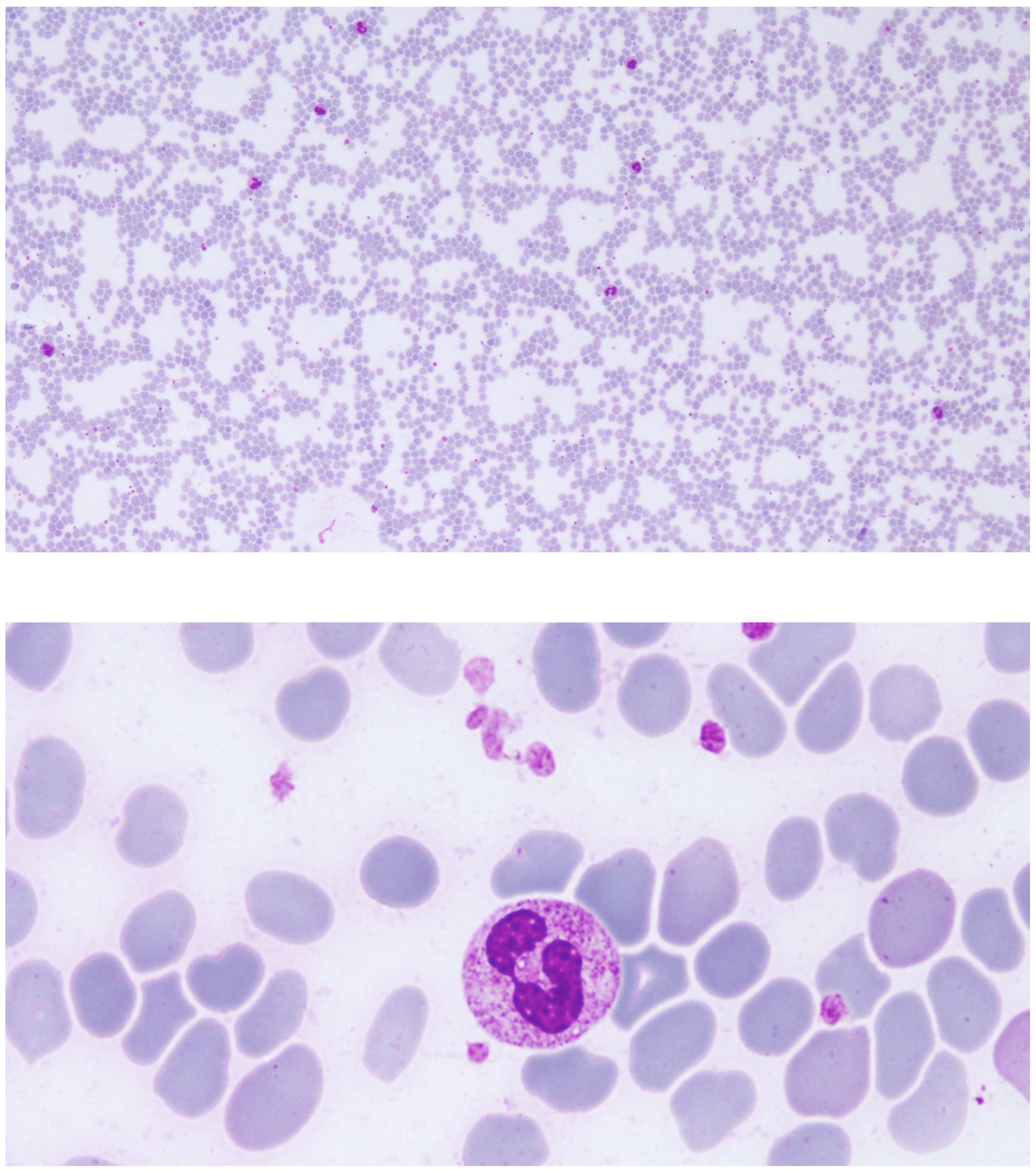

Morphological analysis M- and PMN-MDSC subtypes on blood smear after MayGrünwald-Giemsa. 


\section{Supplemental Figure 3}

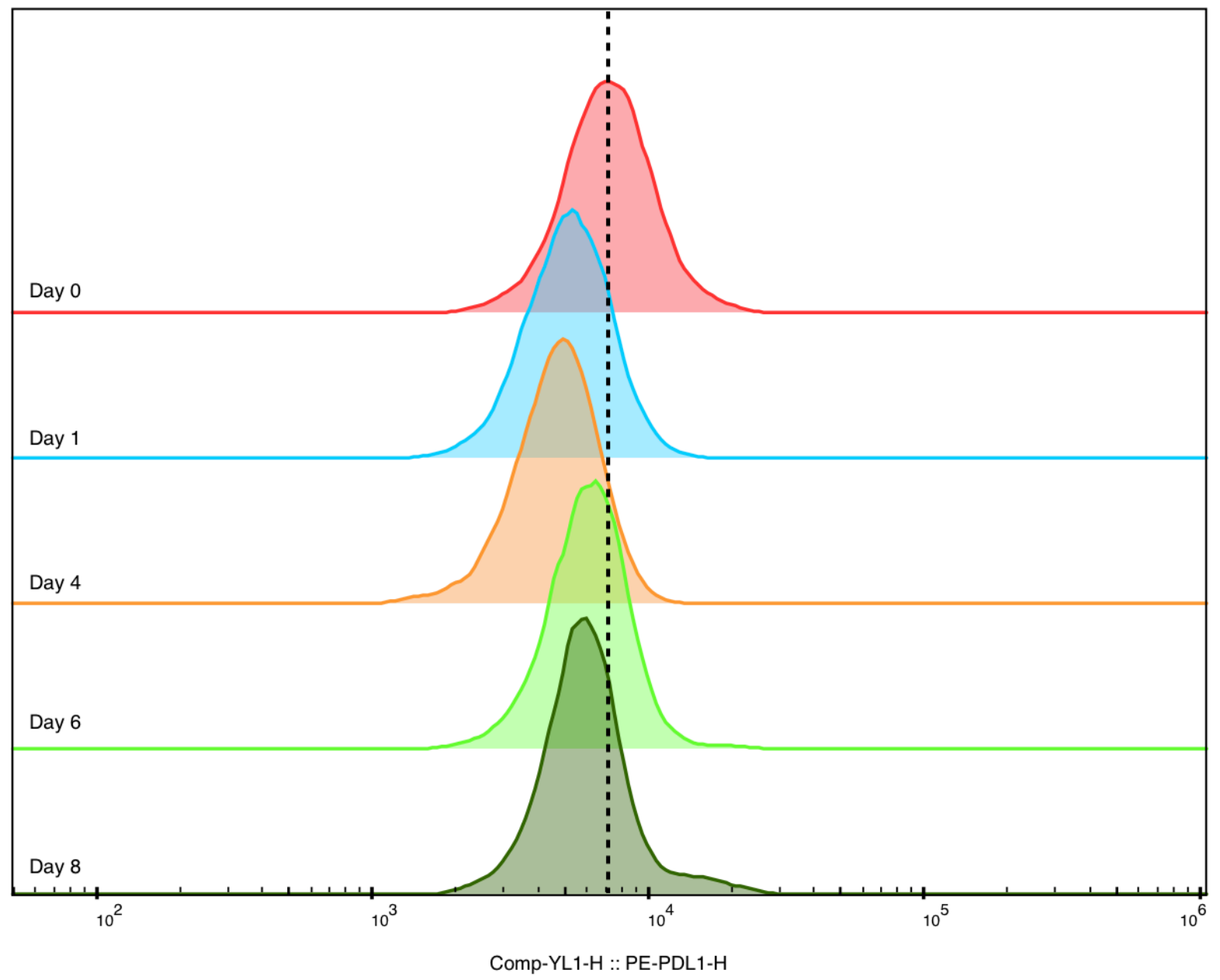

Flow cytometry overlays showing differential PD-L1 expression before (day 0) and after immunotherapy. Stacked histograms were used to enhance data analysis. 


\section{Supplemental Figure 4}

PMA-stimulated cells

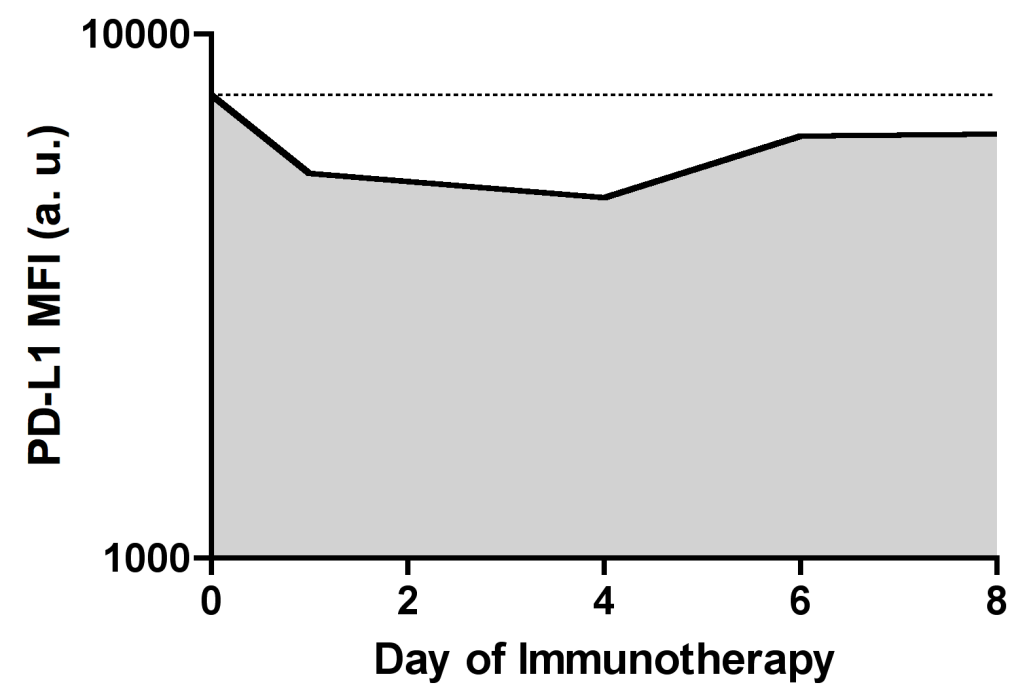

Mean fluorescence intensity (MFI) detected by flow cytometry before and after immunotherapy after exposure to PMA.

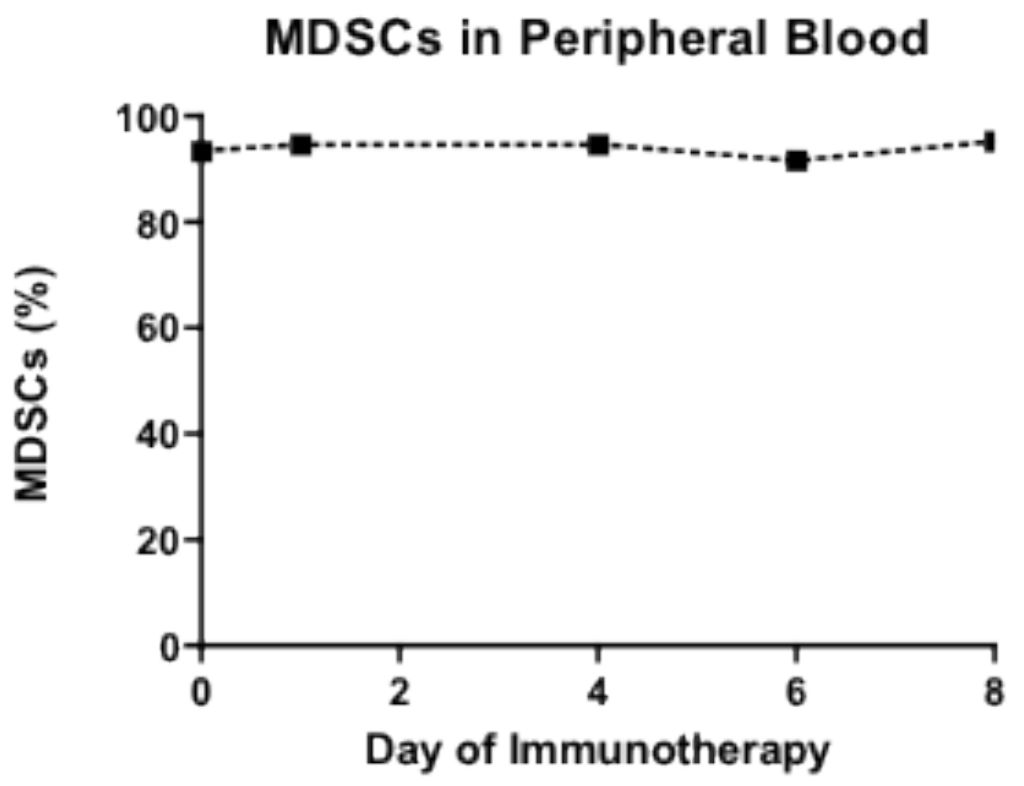

Relative MDSCs detected by flow cytometry before and after immunotherapy after exposure to PMA. 